\title{
(0) Agency, embodiment and enactment in psychosomatic theory and practice \\ OPEN ACCESS
}

\author{
Laurence J Kirmayer, Ana Gómez-Carrillo
}

Psychiatry, McGill University, Montreal, Quebec, Canada

\section{Correspondence to}

Dr Laurence J Kirmayer, Psychiatry, McGill University, Montreal, QC H3A 1A1, Canada; laurence.kirmayer@mcgill.ca

Received 6 November 2018 Revised 14 March 2019 Accepted 19 March 2019 Published Online First 5 June 2019

\section{ABSTRACT}

In this paper, we examine some of the conceptual, pragmatic and moral dilemmas intrinsic to psychosomatic explanation in medicine, psychiatry and psychology. Psychosomatic explanation invokes a social grey zone in which ambiguities and conflicts about agency, causality and moral responsibility abound. This conflict reflects the deep-seated dualism in Western ontology and concepts of personhood that plays out in psychosomatic research, theory and practice. Illnesses that are seen as psychologically mediated tend also to be viewed as less real or legitimate. New forms of this dualism are evident in philosophical attacks on Engel's biopsychosocial approach, which was a mainstay of earlier psychosomatic theory, and in the recent Research Domain Criteria research programme of the US National institute of Mental Health which opts for exclusively biological modes of explanation of illness. We use the example of resignation syndrome among refugee children in Sweden to show how efforts to account for such medically unexplained symptoms raise problems of the ascription of agency. We argue for an integrative multilevel approach that builds on recent work in embodied and enactive cognitive science. On this view, agency can have many fine gradations that emerge through looping effects that link neurophenomenology, narrative practices and cultural affordances in particular social contexts. This multilevel ecosocial view points the way towards a renewed biopsychosocial approach in training and clinical practice that can advance person-centred medicine and psychiatry.

I know so little about the activity of the pineal gland Really, what do I have in common with my body. - Anna Swir

\section{INTRODUCTION}

Psychosomatics is a field born of mind-body dualism. It is precisely the implicit ontology of Western medicine that treats mental and bodily sickness as two very different kinds of entities that warranted the creation of a special field tasked with explaining 'the mysterious leap from the mind to the body'. ${ }^{2}$ From the perspective of other cultural ontologies or medical systems that see psyche and soma as two facets of the same set of processes, there is no leap, no distinctive category of illness, and no special family of psychosomatic mechanisms-though still plenty of mystery as to how social and psychological processes shape experience in health and illness.

Psychosomatic medicine developed as a subspecialty of psychiatry at a time when psychodynamic thinking was dominant in the United States, but has come to serve as an umbrella term for a broad range of research and clinical activities in behavioural medicine, health psychology and consultation-liaison psychiatry. ${ }^{3}$ With the waning of psychodynamic theory and practice in psychiatry, the ways we frame our theoretical puzzles have changed. The challenges today concern brain-gene-environment interactions, epigenetics, neuroplasticity and precision medicine. A large and rapidly growing body of research provides models of disease in terms of multilevel interactional processes. This work makes it clear that both illness experience and the mechanisms of disease are driven not only by pathophysiology, but equally by psychological and social interactional processes. Yet, despite these advances, fundamental tensions remain that are rooted in the sociomoral dimensions of illness experience. These are reflected in the conceptual vocabulary we use in everyday explanations of illness and affliction. These explanations tend to dichotomise experience into events we can control and hence are responsible for and those that are beyond our control—accidents or unfortunate outcomes of forces too large or too small to be within the ambit of human agency. Of course, new knowledge and technologies can extend the reach of agency and change the social, moral and psychological significance of particular forms of affliction. Moreover, there are many potential forms of control and corresponding varieties or gradations of agency. But both folk psychology and practical reasoning in the clinic tend to employ simpler, often dichotomous models in explaining illness and disease.

In this paper, we want to examine some of the forms and consequences of this reasoning about illness causation in clinical practice and then point toward a more biologically, socially and experientially integrative view which has important consequences for research, theory and clinical practice. First, we discuss the ubiquity and persistence of mind-brain dualism in everyday thinking as well as clinical interactions. ${ }^{4}$ We then consider the ways in which the biopsychosocial (BPS) approach introduced in the 1960s aimed to integrate multiple levels of explanation. Next, we review some criticism and concerns about the BPS and its displacement by a 'bio-bio-bio' approach, which focuses exclusively on biological explanation. We then outline how a view rooted in contemporary 4-E cognitive science points toward a renewed BPS approach. This approach is illustrated by a discussion of resignation syndrome (RS) among refugee children in Sweden. We end with some reflections on why it remains difficult to adopt this more integrative view and suggest some ways forward. 


\section{EVERYDAY DUALISMS}

Though most illness experience makes any separation of mind and body seem strange, biomedicine continues to organise its institutions and practices in ways that impose a sharp divide with sometimes profound consequences. Triage in hospital emergency rooms sorts patients out in terms of those with problems that need to be seen by an ER doctor and those who need to be referred to psychiatry. Hospitals maintain consultation-liaison psychiatry services that see patients with concurrent ('comorbid') psychiatric disorders. Patients and their families struggle with the stigma of being told they have a mental health problemwith the implication that the problem is somehow less real, their own fault, or even 'all in your head'. ${ }^{5}$

Although when questioned directly, most clinicians likely would adhere to a monistic model that asserts that all health problems have both physical and mental (psychological, emotional or cognitive) dimensions, there is evidence that even psychiatrists maintain dualistic thinking about everyday clinical situations. ${ }^{6}$ Some problems tend to be viewed as more biological (and hence due to mechanisms beyond the individual's direct control), while other conditions are seen as more psychological or behavioural (and hence, potentially under the individual's conscious control). Problems of substance use or addiction are located somewhere in the middle, reflecting ongoing debates about the nature of habits, but the notion that addictions reflect weakness in character persists. This dichotomy is not confined to medicine but reflects a broader dualistic ontology intrinsic to our concept of the person. This dualistic understanding of the person recognises that there are events we cause through our own deliberate actions and events that just happen to us because of impersonal forces within or outside our brains and bodies. Attempts to circumvent this everyday dualism must contend with the ethical implications of any shift in our notion of personhood.

The mind-body problem, which is reflected in our perplexity about psychosomatic causation, has undergone much refinement and elaboration in the centuries since Descartes. At present, it bundles together three separate problems. ${ }^{7}$ The first concerns how complex cognition and behaviour can emerge from lifeless matter. The second centres on the hard problem of consciousness. The third is concerned with moral questions of agency, responsibility and blame.

The problem of emergence of complex behaviour is the area where significant process has been made through systems science. A hierarchical system's view shows how simple units can be built up to create more complex control systems. Even simple systems, however, can show complex behaviour. The cybernetic models of the 1940s and 1950 s yielded important insights about the ways in which control can go awry. ${ }^{8}$

Systems science shows how complex behaviour can emerge from simple networks with iterative or feedback loops. ${ }^{9}$ Cybernetic theory suggests that goal-directed behaviour arises from networks with appropriate feedback loops, which thus exhibit a kind of intentionality and agency. ${ }^{10}$ In a hierarchy of such control mechanisms, we move from experience-distant, automatic processes like maintaining a comfortable level of strain on a muscle or joint, to higher order goals that must be described in ways that are experience near and narrative based, which may involve striving to adhere to an account of oneself in terms of specific traits, values and reasons that are part of a life story. At this higher level of control, reasons can become causes. Hence, we must recognise the causal efficacy of our own narrative accounts of self and other. ${ }^{11}$
The ways that we narrate our experience influence our interactions with others in the social world and this, in turn, reshapes our experience. These kinds of feedback processes are central to what the philosopher Ian Hacking has called 'looping effects'. ${ }^{12}$ Looping effects can occur at many levels in the hierarchical organisation of biological and social systems, including the internal neural and hormonal regulation of the body's physiological systems; the bodily-cognitive-emotional cycles that contribute to the amplification of distress; sensorimotor engagements with the environment; interpersonal interactions in dyads, families and other social groups; and the larger social processes of institutionalising particular conceptual categories and practices like medical diagnosis. ${ }^{13}$ In his own historical case studies, Hacking shows how looping effects at the broadest conceptual and societal level influence our ontologies. Moreover, looping effects can traverse multiple levels of organisation, linking the different levels (eg, biological and social) needed to understand and explain human experience in health and illness. ${ }^{14}$

Many who concede that complex behaviour and cognition can emerge from suitably arranged computational networks would still insist that the most distinctive features of human experience, our conscious awareness and subjectivity, represent a fundamentally different process that cannot be given a materialist account. This so-called 'hard problem' of consciousness is the last bastion of dualists. ${ }^{15}$ Efforts to explain consciousness may reduce it to capacities for self-observation and narration, link it with specific brain circuitry that enables arousal, activation, attention and awareness, equate it with the overall capacity for multimodal information integration in the brain, or make it intrinsic to sensory-perceptual engagements. ${ }^{16}$ However, these efforts do not satisfy those who see a profound gap between the subjective quality of experience and mechanistic explanations. Although much is made of the difficulty of getting from mechanism to qualia, this conceptual gap is usually of little concern in clinical practice for several reasons: (1) we accept experience as important in itself, whether as an indicator of past or present events or of the ways in which the patient as person understands and endures affliction, pain and suffering; (2) pain and pleasure (and other valenced experiences) provide an essential dimension in judgements about the value, consequences and moral significance of action; and (3) individual experience is central to the social presence and personhood of the other as subject and agent in moral decision-making.

Whatever the fate of unifying or mechanistic accounts of consciousness, there is a residual form of mind-body dualism that is recalcitrant and persistent in our thinking because it reflects notions of agency that are basic to moral judgements of responsibility and blame. Simply put, there are events we cause through intentional actions and those that just happen to us. We are held accountable for the former in everyday moral and juridical contexts; the latter we are excused. Indeed, the notion of the sick role builds on the sense that illness is misfortune that befalls us. Of course, in practice, there is a very wide grey zone which includes lifestyle choices, habits, akrasias and errors of judgement that lead to affliction (obesity, alcoholism, addictions, injuries due to risky behaviour, etc.). The scope of this grey zone and the shades of grey or distinctions within it depend on local social and cultural norms.

The residual form of dualism is linked to our embodied sense of ownership and agency, giving rise to an implicit ontology. From an early age, we tend to consider things that move on their own as animated (anima: breath, soul, spirit, vital force) and to ascribe agency to these animate beings. ${ }^{17}$ Agency is then metaphorically extended to more abstract concepts, including 
the self as an agent to underwrite ascriptions of moral responsibility. ${ }^{18}$ We also learn early on to ascribe agency to our intentions rather than our actions, when the actions do not correspond to our intentions. Thus, a child might explain that she does not want to cry but her body does. The embodied experience of crying then conflicts with the intention to not cry. Indeed, as the epigraph to this paper notes, most bodily processes, including emotional experience and expression, occur through subpersonal mechanisms to which we have no direct access. This gap between mechanism and intention gives rise to the experiential tension or contradictions that fuel our dualistic ontology of mind and body. ${ }^{19}$ Arguably, this tension represents a universal existential predicament, which every culture develops conceptual resources and strategies to manage ${ }^{20}$. Indeed, this tension ensures that no matter how clearly we articulate holistic, integrative mechanistic theories of illness, patients and clinicians will continue to struggle with the social and moral meanings of affliction.

There are also cultural or religious variations in ontologies that can construe any event as a result of our own moral actions, failings or transgressions (through karma or divine justice), as caused by others' actions, or as the inevitable outcome of a chaotic and capricious universe. For example, many African religious ontologies provide explanations of affliction in terms of moral transgressions involving spirits, ancestors and envious neighbours. ${ }^{21}$ There is always some external malign agency available to explain suffering. Hence, any affliction can be viewed as caused by a conjunction of human ill will and socio-spiritual agency. The implications of this external attribution of agency must be understood in terms of the dynamics of collectivist societies. In contemporary Western individualistic societies, stress plays a similar role, providing a universally applicable explanation that conjoins individual human agency and structures or arrangements beyond individual control (society, the economy or 'the system'), but always with the implication that illness can be avoided by those who are more resilient and better able to manage the stress of life. ${ }^{22}$ This means that behind even holistic or totalising ontologies, there is plenty of room for everyday negotiations that assign causality, control, responsibility and blame in ways that are enabling or disqualifying for individuals. ${ }^{23}$

Biomedicine aims to develop objective markers of disease process that can circumvent the unreliability of self-reports. The emphasis on biological parameters is underwritten by an epistemic dualism that sharply distinguishes objective physical signs from subjective (psychological) complaints and that, in turn, supports the ontological distinction between 'real' bodily illness and 'imaginary' psychological distress. ${ }^{24}$ The lack of biomarkers for many conditions then threatens the 'objective' reality of illness. This is evident in the special stigma experienced by patient's with medically unexplained or contested symptoms and syndromes. ${ }^{25}$

Of course, the very lack of biomarkers for psychological processes allows them to be used with great flexibility in constructing explanations. Just as stress is always available as an explanation for affliction, psychological factors can be invoked to account for both the occurrence and the symptomatology of any affliction. In the early years of the 20th century, the internist-psychiatrist Georg Groddeck elaborated a model in which every affliction could be treated as a result of the logic of the 'It'. ${ }^{26}$ Ironically, modern self-help and positive psychology approaches at times embrace the same totalising mode of explanation, although in settings that may offer social support and helpful coping strategies. ${ }^{27}$ While this kind of explanation offers meaning and the promise of agency in mastering illness, it also may entangle sufferers in a web of moral culpability that aggravates their condition.

In sum, although we can distinguish ontological, epistemic and moral versions of dualism, all three are at play in biomedicine and serve to rationalise, stabilise and reinforce each other as a tacit social logic and institutional practice. Hence, scientific advances or changes in clinical practice that alter the terms of debate about one form of dualism may be resisted or undermined by the persistence of other forms.

\section{EXPLANATORY PLURALISM: THE BIOPSYCHOSOCIAL APPROACH}

A major effort within biomedicine to transcend conceptual dualisms took the form of the BPS approach elaborated by internist-psychoanalyst George Engel in the late 1960s and 1970s. Engel proposed the BPS model as a response to several concerns in the medicine and psychiatry of the time. ${ }^{28}$ One was to protect a place for subjectivity in the clinical encounter, in the face of increasingly mechanistic accounts of pathology. ${ }^{29}$ In this, Engel was influenced by Adolf Meyer who argued, as one follower put it, that ' $[t]$ he patient comes with his own view of his trouble; the physician has another view. Treatment consists of the joint effort to bring about that approximation of those views which will be the most effective and the most satisfying in the situation'. ${ }^{30}$ In the clinical encounter, then, subjectivity is the primary ground of meaning and agency for the patient and, hence, essential to understanding and responding effectively to suffering.

Meyer's perspective left a gap between experience (symptoms and illness) and mechanism (pathophysiology and disease) that psychodynamic theory could not resolve. Borrowing from general systems theory, Engel offered a more integrative view based on the recognition that nature is organised in terms of hierarchical systems with each level in the hierarchy having its own emergent dynamics. ${ }^{31}$ The hierarchical systems view of the BPS was presented as a pragmatic and programmatic answer to the ontological and epistemic dualisms inherent in biomedical theory and practice. ${ }^{32}$

Engel's approach became a mainstay of medical education, providing a framework for the multiple languages of description, and modes of causal explanation that constitute the basic science curriculum. ${ }^{33}$ However, despite its wide acceptance, the BPS approach has also received some criticism. Notably, the psychiatrist and philosopher Nassir Ghaemi critiqued the BPS approach, arguing that it promoted an unprincipled eclecticism that failed to integrate models grounded in evidence. ${ }^{34}$ To Ghaemi, it seems that only biology can provide scientifically sound modes of explanation for psychiatry. An adequate conceptual framework for research and practice should therefore be based entirely on neurobiology, complemented by phenomenology, to allow the clinician to understand and engage the patient's lifeworld.

While Ghaemi's critique of the BPS model has many failings, it is revealing of the crisis in American psychiatry that occurred from the 1980s onward with the loss of psychodynamics as a dominant paradigm. ${ }^{35}$ The displacement or demotion of psychoanalysis, which had been central to psychiatric education, left an enormous gap in the training of psychiatrists who had little exposure to academic psychology or social sciences. This intellectual vacuum was filled by neuroscience. As a result, while the BPS allowed for-indeed, encouraged-explanatory pluralism, psychiatry has taken a different turn, embracing the primacy of neurobiological explanation in what has been characterised as a 'bio-bio-bio' approach. ${ }^{36}$ This emphasis on biological explanation is evident throughout contemporary psychiatry, which has 
invested heavily in the notion that neuroscience will (eventually) provide adequate explanations for mental disorders.

One expression of this view is the Research Domain Criteria (RDoC) framework produced by the US National Institute of Mental Health to guide research on mental disorders. ${ }^{37}$ Presented as a $2 \times 2$ matrix of biobehavioural systems and levels of analysis, the RDoC scheme incorporates a rudimentary BPS structure, explicitly acknowledging molecular (genetic), cellular, physiological and circuitry levels of analysis. However, higher levels are given short shrift, with the whole of subjectivity, narrativity and intersubjectivity collapsed into a level tersely labelled 'self-report'. The RDoC framework privileges the level of neural circuitry on the assumption this is the level of organisation at which the disturbances of function that characterise psychiatric disorders will eventually be explained. Although the RDoC framework includes some distinctively human cognitive and social systems, and it remains open to adding new dimensions, the framework clearly emphasises those systems that can be studied through animal models. Unfortunately, there are no mouse models of narrativity, poetics or storytelling, nor of the existential confrontation with mortality, racism, political violence, forced migration and transnational adoption-to name only a few of the common social predicaments that contribute to mental health and illness. ${ }^{38}$

\section{PHENOMENOLOGY IN CONTEXT: 4-E COGNITIVE SCIENCE, AGENCY AND LOOPING EFFECTS}

Clinicians who embrace neurobiological explanations of mental disorders may nevertheless acknowledge the value of phenomenology both to make diagnostically relevant distinctions and to understand and engage patients. While phenomenology offers a corrective to the absent person in neuroreductionism, it has undergone a strange truncation in North American psychiatry. In place of close attention to the structure of experience, from DSM-III onward, 'phenomenology' has come to refer to lists of symptoms enshrined in diagnostic criteria and research checklists. ${ }^{39}$ In this technology, there is little attention to the quality of patients' experience, its structure, meaning and significance in context.

European psychiatry has maintained an interest in more systematic study of psychopathological experience, building on the tradition of phenomenological psychiatry that goes back to Karl Jaspers. ${ }^{40}$ However, most of this work still tends to bracket off or even ignore context as a crucial determinant of both the content and structure of experience. The relevant contexts for psychiatry include the body, the individual's personal history or biography, their family and community, local cultural world and social position, as well as larger societal, national and transnational networks, institutions and discourses. Serious consideration of context reveals that the social world not only shapes but is actually constitutive of experience. ${ }^{41}$

The challenge that remains for medicine and psychiatry is how to bring together the experience-near understanding of illness experience with the detailed, multilevel explanation in terms of biological, psychological and social processes promised by the BPS approach. A bridge between phenomenology and mechanistic explanation can be found in the growing body of work in 4-E cognitive science, which emphasises the embodied, embedded, enactive and extended nature of cognitive processes. ${ }^{42}$ Contemporary theories of embodiment and enactment provide a framework for understanding the interplay between bodily experience and the environment. ${ }^{43}$ In this view, perception and action occur in and with the body. This always occurs through interactions with a culturally shaped social environment that affords specific modes of action. ${ }^{44}$ Developmentally early sensorimotor processes serve as a basis for the scaffolding for more complex metaphoric and abstract cognition. ${ }^{44}$ Even abstract cognition therefore is embodied in that it emerges developmental from bodily experiences and retains dynamic links to bodily processes. The process of engaging and extending mind in a particular niche brings into being (enacts) particular modes of relatedness and, in so doing, reconfigures the physical and social arrangements with which we scaffold our experience. ${ }^{45}$ Looping effects between brain, body and environment underlie the process of embodying, enacting and extending cognition and experience across the different contexts of embedding (body, self, dyad, family, network, society). Applying the 4-E perspective to symptom experience allows us to move beyond dualism toward an ecosocial view of the co-constitution of mind, brain and body in health and illness. This perspective also has implications for our notions of agency and causality.

Agency involves the sense of ownership, causality, control and responsibility for actions which are related to our goals, plans and intentions. The 4-E perspective sees agency as arising from loops of action and perception. ${ }^{46}$ These loops may result in certain actions being interpreted as agentic because we experience the covariation of our attention, goals, actions and sensations in real time, particularly when we shift our actions in response to a change in goals or intention. ${ }^{47}$ The dynamics of these action-perception loops then provide a basic sense of ownership and agency, but the experience and ascription of agency also depends on the interpretive frameworks we bring to bear. ${ }^{48}$ Moreover, these action loops can extend into the social world to include others who may be part of the process of experiencing agency. Causality and control need not be aligned. Cause may involve a long chain of events including the actions of others, our own previous actions, and features of the acting body and its current context. Control may also reflect multiple processes that are hierarchically structured, with lower-level processes that are more or less automatic (reflexes, habits), and higher-level choices or decision-making that may involve conscious deliberation or consultation with others. ${ }^{49}$.

Still finer gradations of agency can be distinguished by considering: (1) multiple (top-down cognitive) controls in action; (2) the intervening functions of affective dispositions and emotions; (3) the affordances and constraints of contexts (norms, expectations, elicitations or invitations), including forms of instrumentality and discursive possibilities; (4) the consequences of actions for social positioning and the responses of others; and (5) the wider social, economic and political processes that give individuals or groups voice, power, resources and authority. Each of these gives rise to alterations in the experience of agency-each can support and stabilise or fractionate and disrupt the other. This gives rise to a rich set of potential distinctions for human agency. Unfortunately, we lack clinical language for these distinctions and our everyday thinking confounds causality and control so that, in practice, we usually fall back on a simple dichotomy: wilful or not. This has important consequences for the ways we think about causality, responsibility and blame in health and illness.

\section{THE EXAMPLE OF RESIGNATION SYNDROME}

The strange story of apathetic syndrome in Sweden provides an instructive example of the problem of agency in psychosomatic explanation. Since 2001, several hundred cases of refugee children have presented clinically with a syndrome 
Table 1 Modes of explanation of resignation syndrome

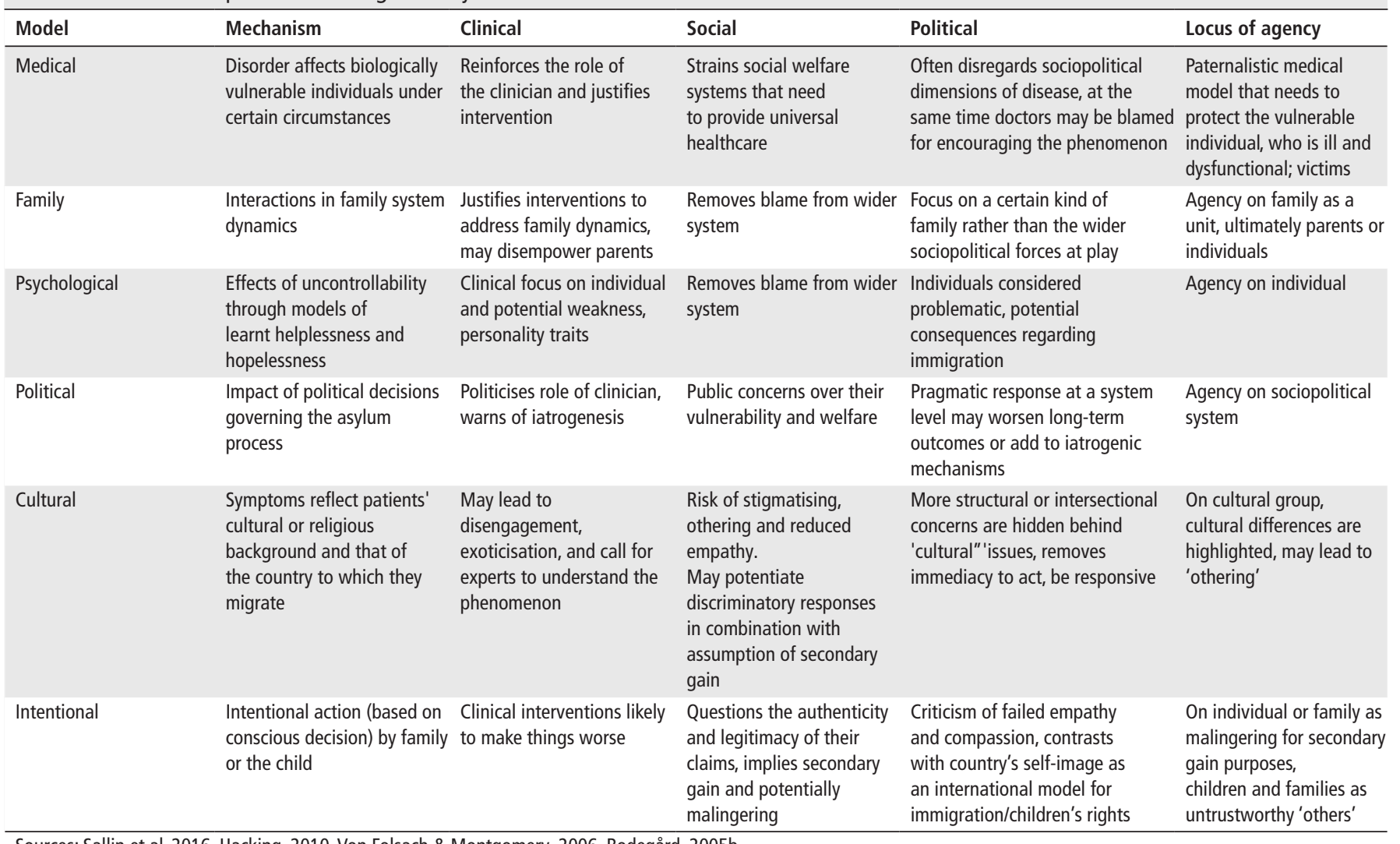

Sources: Sallin et al. 2016, Hacking, 2010, Von Folsach \& Montgomery, 2006, Bodegård, 2005b.

characterised by depression and behavioural withdrawal that progresses to stupor and finally a state of apparent unconsciousness, with loss of pain response, requiring nasogastric tube feeding and intensive care. ${ }^{50}$ This condition, reported exclusively in Sweden, has been termed 'apathetic' or 'uppgivenheits syndrome'-literally, 'given-up syndrome' but officially translated as 'resignation syndrome'. The disorder occurred among children whose families were stuck in 'limbo' in the process of migration facing an uncertain outcome. Recovery could take months to years and appeared to be related to the restoration of hope to the family, often through receiving permanent residency.

As the number of affected refugee children increased, an official inquiry and expert committee were convened to help reach consensus on diagnosis and management. ${ }^{51}$ The Swedish National Board of Health and Welfare officially recognised RS as a novel diagnostic entity, with ICD-10 code F32.3A which locates it within the affective disorders, with the added specifier Z65.8A 'problem adhering to status as refugee and asylum seeking. ${ }^{, 52}$

As part of their report, the expert committee provided the six etiological models we have listed in Table 1 . The phenomenon was explained variously in terms of neurobiological, psychological affective, stress-related, social-systemic, cultural, political mechanisms and intentional actions. Other explanatory models included psychodynamic perspectives, that invoke notions such as 'lethal mothering' or trauma reenactment, as well as the notion of 'resistance through regression' seen as part of a refusal-withdrawal-regression spectrum. ${ }^{53}$ While all the elements of the BPS model can be found distributed across these accounts, they remained unintegrated in the contentious debate between those proposing psychogenic mechanisms, those proposing social causation models, and those struggling to describe neurobiological mechanisms, correlates and signs that would provide an 'objective' diagnosis. Each account was criticised for its biases or shortcomings. Sociologists were accused of a lack of compassion because they related the phenomenon to collective social processes, while clinicians who argued for the neurobiological basis of the syndrome were accused of encouraging or maintaining the problem, if not actually causing it.

To understand what is at stake in these criticisms, it is instructive to consider the work that each of these explanatory models does from a clinical, social and political perspective, especially with regard to where they locate agency. The location of agency has implications for how symptoms may function as an idiom of distress, the processes that underlie the social course of illness, and the development of strategies for treatment and prevention.

The medical accounts mainly relied on variations of the diathesis-stress model, listing a series of predisposing, precipitating and perpetuating factors. The family model drew elements from both family systems and psychodynamic theories, focusing on the role of dysfunctional family dynamics described as overprotective, overanxious and mistrusting parents with difficulties in setting limits and in tolerating separateness. ${ }^{54}$ The family model thus emphasised how parents' health and stability affected the child, and noted parental mistrust of the medical system when medical evidence revealed no organic cause for the child's symptoms.

The psychological model focused on personality traits of the children (described as perfectionist, conscientious, high achievers) and their history of emotional or behavioural disturbances, such as aggressive and sexually provocative behaviour, 
separation anxiety, school refusal and somatisation or dissociative disorders. ${ }^{55}$ The political model took a more systemic view and focused on the role of the asylum process and the health systems response to RS in aggravating the problem. ${ }^{56}$ Media discussions of the time reflected on the political context and social tensions that shaped the environment in which the syndrome emerged. ${ }^{57}$

The cultural explanations used a relatively narrow approach to culture that used the notion of a 'culture-bound syndrome' or made vague references to cultural beliefs, ethnic-specific genetic or epigenetic features to explain why RS was confined to Sweden and to particular ethnic or linguistic communities. ${ }^{58}$ Some accounts invoked stereotypes of childrearing among the ethnic or language minorities most affected.

The intentional model viewed the symptoms as deliberate behaviour and invoked secondary gain from the illness as the underlying causal mechanism, suggesting that having a severe illness increased the likelihood of asylum approval. ${ }^{59}$ This model was reflected in the emergence of rumours that the children were taking drugs or being drugged by their parents to produce the symptoms of RS, as well as in allusions to chronic factitious illness or Munchhausen by proxy. ${ }^{60}$ Some authors noted that withdrawal and apparent unconsciousness could also provide the children with the primary gain of relief from the reality of their family's social predicament. ${ }^{61}$

Each approach to explaining the syndrome raised its own questions about clinical epistemology (ie, how to validate diagnostic explanation with the kinds of knowledge available in clinical contexts), intervention and prevention. ${ }^{62}$ The struggle to validate and explain the syndrome also revealed important social and political dynamics related to the local and global refugee crisis, the path to permanent resident status, the rising costs to the healthcare system, and the threat to Sweden's image as a welcoming place for refugees.

\section{WHAT'S IN A NAME? THE DYNAMICS OF DIAGNOSTIC PRACTICE}

The story of uppgivenhetssyndrom is a clear example of the role of the social, political and economic processes in shaping illness experience and in the way local diagnostic practices validate certain symptoms and syndromes. Acknowledging this context should prompt a discussion of the clinical, social and moral consequences of diagnostic labelling. For our present purposes, we are mainly concerned with the ways in which clinical diagnosis and formulation make explicit or implicit attributions of agency that recognise or obscure the sociocultural processes at play across the hierarchical levels of organisation relevant to human experience.

Practically, a diagnosis is legitimated when clinicians adopt it, based on their acceptance of the epistemic authority of nosological systems, official guidelines or local communities of practice. In the absence of a formal diagnostic label, the availability of specific biological markers that can be measured might also validate a symptom or syndrome but, at present, this is not available for most psychiatric disorders. In the absence of discrete diagnostic categories and biomarkers, research evidence of treatment efficacy can also legitimate diagnostic entities by linking them to differential therapeutics and a plan of action, which can become accepted clinical practice and, especially when disseminated through mass media and the internet, come to drive patients' expectations. These are all paths through which diagnostic entities come into being as available labels for certain kinds of experiences and can then participate in looping. ${ }^{63}$

Clinicians' use of a diagnosis and the symptoms subsumed under it creates a pool of socially legitimated idioms of distress that influence illness experience and that individuals can draw from to make sense of and communicate their distress. ${ }^{64}$ Diagnostic codes take on added significance as part of the administrative allocation of services in healthcare systems, insurance reimbursement, and other social welfare or immigration systems. Repeated use of a diagnostic term in any of these contexts serves to reify, legitimate and stabilise the entity it names.

At the same time, locating a diagnosis within psychiatric nosology creates a dichotomy between those disorders that may be considered 'psychogenic' versus those that are viewed as 'organic'. This means that even while the diagnostic entity is being legitimated as a formal part of diagnostic practice, it is assigned an ambiguous, ambivalent or negative moral status. The problems with this status can be seen in the resistance of patients with chronic fatigue to any psychiatric or psychological description and treatment or in the efforts of advocacy groups to avoid classifying autism as a psychiatric disorder. ${ }^{65}$

The various diagnostic labels suggested for the Swedish refugee children's syndrome exemplify this dilemma. Depressive devitalisation (DD) and pervasive refusal syndrome (PRS) followed a psychological explanatory model and differed mainly in that PRS was considered active refusal by the children and did not include 'flaccid paralysis and generalised sensory loss'. ${ }^{66}$ These two labels clearly would have very different consequences for treatment and provoke distinctive social and political responses than the proposed alternative, pervasive arousal-withdrawal syndrome (PAWS). The diagnosis of PAWS introduced an explanation in terms of a neurobiological mechanism of simultaneous hyper-arousal of the sympathetic and parasympathetic autonomic nervous systems, which results in a 'deadlock' that manifests as refusal. ${ }^{67}$ This description aimed to account for the same symptomatology as the above-mentioned DD or PRS but re-conceptualised 'extreme anxiety avoidance' and 'behavioural paralysis' as expressions of the specific patterns of autonomic response.

While the term 'psychogenic' used in reference to etiological mechanisms is contrasted with somatic, physical or organic, and hence reflects persistent dualistic thinking, the problem lies less with this ontological distinction than with the implications for issues of agency, moral judgement and blame that follow from this label. This is evident in the observation that when using the adjective 'psychogenic' in discussions of RS, many authors immediately clarified that by this they did not mean consciously provoked, on purpose or invented.

This dilemma can be seen by comparing the language used for PRS ('symptoms of extreme social withdrawal and determined refusal to walk, talk, eat, drink or care for themselves in anyway') with that of DD, which describes symptoms in terms of psychodynamic explanations of troubled motherchild relations in severely traumatised families. ${ }^{68}$ The diagnostic labels themselves even hint at where agency is being located-'refusal' versus 'devitalisation', which points towards another label used to describe RS in Sweden, 'apatisk' (literally, apathetic). However, the language of PAWS focuses on a neurophysiological account of the refusal behaviour paired with high energy consumption and brain activity shifts. Another example of subtle agentic ascription through language choice occurred when clinicians described the phenomenon as a syndrome, while some social theorists rejected this medical 
term in favour of 'behaviour' ${ }^{69}$ Although framed as clinical or scientific issues, each of the explanations tendered depended on specific notions of causal mechanism and agency with potential social and political consequences.

\section{THE SOCIAL LIFE OF SYNDROMES}

RS arose in the context of increasing social tension about migration, with heightened fear and caution towards or even rejection of refugees in many nations that have historically viewed themselves committed to a positive reception to asylum seekers. In the midst of political turmoil and media reports that constructed a narrative of 'how obliging politicians, unsuspecting journalists, organised right-wing extremist, power doctors and psychiatrists create an image of the so-called "apathetic children"', ${ }^{70}$ in a country that had long viewed itself as an international model for immigration and children's rights, it is not surprising to find a vigorous effort to find neurobiological explanations for such alarming symptoms. From PAWS to 'psychogenic catatonia' based on altered brain metabolism, accounts faced the imperative to clarify the validity of the symptoms against the assumption of intentionality and secondary gain. The choice was framed in stark and more subtle or technical terms: apathy versus refusal; disorder versus manipulation; biological versus mental; syndrome versus behaviour.

Resignation syndrome arose in the context of increasing social-tension about migration, with heightened fear and caution toward or even rejection of refugees in many nations that have historically viewed themselves committed to a positive reception to asylum seekers. In the midst of political turmoil and media reports that constructed a narrative of "how obliging politicians, unsuspecting journalists, organised right-wing extremist, power doctors and psychiatrists create an image of the so-called "apathetic children" ${ }^{70}$ in a country that had long viewed itself as an international model for immigration and children's rights - it is not surprising to find a vigorous effort to find neurobiological explanations for such alarming symptoms. From the PAWS, to 'psychogenic catatonia' based on altered brain metabolismaccounts faced the imperative to clarify the validity of the symptoms against the assumption of intentionality and secondary gain. The choice was framed in both stark and more subtle or technical terms: apathy versus refusal; disorder versus manipulation; biological vs mental; syndrome versus behaviour.

This struggle to explain RS reveals some of the tensions and contradictions that continue to trouble psychiatry. Notwithstanding the conceptual advances in medicine, neuroscience and psychology, RS elicited doubt, contestation and a struggle for legitimation similar to that of other medically unexplained symptoms and syndromes (which, like the antiquated construct of hysteria, now fall under the somatic symptom disorder in DSM-5 or bodily distress disorder in ICD-11). ${ }^{71}$. This reflects broader tensions in the notion of psychosomatic explanation, seen in debates over the appropriate response to medically unexplained symptoms. In each case, invoking psychological, behavioural or social explanations is viewed as questioning the validity of distress and not according it the same seriousness and attention as 'bona fide' medical illness. Real distress needs a physiological explanation and a biomarker. The lack of medical validation then results in social stigma and added suffering. Bodegard pointed to this when he warned that RS symptoms may be aggravated and become chronic in response to a 'questioning attitude' within the healthcare system and suggested that such doubt might constitute a form of 'retraumatisation' contributing to the persistence of the syndrome. ${ }^{72}$
Everyday moral evaluations are based, in part, on to whom something is happening (ascriptions of ownership) and who has acted in ways that caused the problem (agency). The ability to make these attributions has a developmental history and emerges first as a form of naïve or intuitive physics and biology (eg, a child's ability to determine what objects are viewed as living organisms capable of initiating their own actions), but is shaped by cultural models and social contingencies and elaborated as a narrative practice, in which one learns to give accounts of the reasons and causes of one's own and others' behaviour ${ }^{73}$.

Whatever causal model of RS one adopts, it is not hard to imagine how the sense of ownership and sense of agency may be challenged and disrupted in the process of migration for the refugee children in Sweden. The uncertainty that dominates their existential predicament, the helplessness and loss of sense of control, likely translate into feeling that no action will restore control, and this sense of powerlessness could contribute to their profound withdrawal. ${ }^{74}$

The story of RS points to the need for conceptual refinements in our understanding of agency, to move beyond the dichotomous thinking imposed by the enduring mind-brain dualism. A more nuanced understanding of agency could show a way beyond the dichotomy of lived experience versus de-authorising scientific materialism. In particular, an ecosocial view allows us to identify multiple forms of agency based on modes of engagement between individual and environment that can be elaborated into a typology of looping effects involving material and discursive features of the world. ${ }^{75}$ This has implications for diagnostic assessment, treatment and prevention.

\section{AN ECOSOCIAL VIEW OF RESIGNATION SYNDROME}

We suggest that RS remains poorly understood because of the lack of detailed models of integrative, multilevel explanation in diagnostic formulation. No single model manages to account for the symptomatology and experience of those affected. The BPS approach is a placeholder for a multilevel formulation but requires further specification to deliver on its promise. Taken seriously, this perspective challenges narrow biological or psychological theories of the self that fail to consider the embedding of all experience in local social worlds. Advances in 4-E cognitive science offer us resources to begin to flesh out this approach and rethink the models implicit in mainstream psychiatry, which were on display in the contentious debate that followed the emergence of RS in Sweden.

An adequate understanding of any form of psychopathology will require an ecosocial perspective that integrates neurobiology and individual experience with broader social and cultural contexts across time. ${ }^{76}$ The social and clinical response to the RS phenomenon were recognised as part of the problem, described as a 'perpetuating' factor that could therefore hold the key to its resolution. ${ }^{77}$ Unpacking the looping effects involved in the chronicity of symptoms requires identifying the social and clinical responses that amplify or exacerbate the RS phenomenon. Such looping effects are not unique to RS and a structured ecosocial analysis of interactions in local niches (families, communities, healthcare systems) can facilitate this unpacking. ${ }^{78}$

A good start on this is the social psychological account of RS offered by Hacking. ${ }^{79}$ Drawing on his earlier work on amnestic fugue as a 'transient mental disorder' in France in the late 1800 s, he proposed an 'imitation and internalisation"'model based on the idea that following the first cases, high levels of media coverage and social cohesion among refugee groups 
may have led to a process of imitation among children with a shared predicament. In a second step, what started as imitation was internalised to the extent that it became an integral part of the child's psychophysical and social constitution through processes of embodiment and enactment. This account is consistent with Shorter's argument that every historical context makes certain symptoms salient and encourages mimetic uptake both by presenting vivid models with which individuals can identify, consciously and non-consciously, and by ratifying experiences through various social practices, including specific help-seeking pathways, modes of diagnosis, intervention and compensation. ${ }^{80}$

This would encourage us to look in two main directions for explanations: inwardly into the biopsychological mechanisms of mimesis, and outwardly toward the social, moral, economic and political embedding (which includes causes and consequences) of illness. The inward processes explain how a mode of illness experience and its local interpretation become installed or embodied in individuals, through processes of 'bio-looping' that link bodily enactment and physiology. The sociopolitical processes explain how it becomes institutionalised. Together these can explain how a new kind of affliction spreads.

This model can be refined by elaborating a hierarchical multilevel BPS approach that integrates illness narratives, embodied neurophenomenology and mechanistic models with ecosocial system thinking. ${ }^{81}$ This would allow us to consider multiple kinds of causal influence and agency arising from loops at different levels, providing a more nuanced account of the impact of structural social and political forces needed to appreciate the practical and moral significance of clinical diagnoses and interventions.

Understanding the embedding of the brain in the social world requires a detailed mechanistic account of the interactive loops between body, person and environment, niche or lifeworld. Computational neuroscience provides a general model of this interaction in terms of predictive processing. ${ }^{82}$ On this view, the brain is constantly generating and updating models that predict sensory input from different modalities and sources. Using Bayesian inference, the comparison between prediction (or prior) and input (from sensorimotor and interoceptive processes or lower-level processing), yields a prediction error. The more accurate predictions are regarding sensory input, the smaller the prediction error. ${ }^{83}$ These predictions are hierarchically structured and can be thought of as encoding plans, beliefs and expectations in top-down models of the local environment, niche or world. Perception is therefore viewed as an active process of inference, through which the model guides what and how sensory input is processed so as to minimise prediction errors. ${ }^{84}$ Alterations in this process can then give rise to various forms of psychopathology. ${ }^{85}$

Sallin and colleagues draw on predictive processing theory to propose a more integrative neurobiological model of the phenomenon of RS. ${ }^{86}$ In their model, a multitude of factorspsychological or physical trauma, helplessness and hopelessness, familial expectations and obligations, the predicaments of migration and asylum seeking-change the priors of the affected child's brain at multiple hierarchical levels following the principle of prediction error minimisation. In effect:

'RS is a condition in which predisposing and contextual factors generate negative expectations and beliefs that are instantiated in fixed prediction priors, which drive homeostatic and behavioural effects as well as self-perception, towards the prediction. This minimises pre- diction error at the cost of pushing the physiological, cognitive and emotional state further away from that which sustains life' ${ }^{87}$

Error minimisation in this case implies that the children's priors across hierarchical levels of organisation are shifted in ways that reflect their ongoing experience of being stuck in an unchanging and unchangeable predicament. The constantly generated (conscious or non-conscious) priors or prediction models of a stressful but static situation, in turn, affect bodily function, for example through alteration of interoceptive regulation of the autonomic nervous system. ${ }^{88}$ As these negative predictions generalise across higher and lower levels of the processing hierarchy in vulnerable individuals, the individual exhibits behaviours such as apathy, withdrawal or catatonia, which elicit support from his or her surroundings.

Although the predictive processing approach offers a conceptual basis for a unified account of the RS phenomenon, current applications treat social and cultural context in a generic fashion without characterising the structure and dynamics that shape and constrain illness experience. A fuller account needs to consider the individual's embedding in hierarchically nested social-cultural contexts, which include the migration trajectory, healthcare system and political conflicts. This embedding can be understood in terms of cultural affordances, that is, the possibilities for interaction with a particular social context or eco-social niche from which an individual's experience, intentionality and action-readiness emerge. ${ }^{89}$ Experience is always preceded by and embedded in cultural systems of meanings and practices, which shape modes of attention and action, as well as interpretive frames and discursive practices that have causal effects as part of hierarchically nested loops linking social position, cognition and bodily processes. ${ }^{90}$ This complicates the medical semiotics that views symptom reports as more or less direct indicators of internal physiological events. Instead, symptom experience must be understood as the outcome of processes of embodiment and enactment, involving discursive practices and negotiations with others in a local social world.

While general processes can be identified, in any actual instance of psychopathology, locally variable modes of interpretation and expression must be considered to give an account of symptom dynamics. This inter-dependence of physiological, psychological and social process follows from the fact that, ontogenetically, humans develop the ability to make sense of and respond to their environments by drawing from inter-subjective processes of embodied experience, social enactment and narrative practices.

If symptom experience, interpretation, expression and response are all influenced by culture and context then culturally shaped coping strategies may change the course of the illness, amplifying or reducing symptoms, distress and functioning. Cognitive-interpretive and social-interactional processes are part of feedback or 'looping' effects that play decisive roles in determining which experiences become symptoms and which symptoms become persistent, distressing and disabling mental health problems. These looping effects occur at multiple levels through intrapersonal, interpersonal, family and wider network or system social processes and determine the causes, course and outcomes of distress. Hence, sociocultural and interpersonal processes play essential roles in resilience and recovery.

Following the trajectory of a refugee child considering these different levels of description and taking an eco-social approach, one can construct the following account. Uprooting and abandoning 'home' is paired with a promise, explicit or implicit, of a better and brighter future, or simply some safety, security and 
well-being. On arrival to a host country, there may be excitement and an effort to make it 'home'. This adaption is often easier for the young than for their parents or elders, which may parentify the children. Concerned about their parents' well-being, children take on tasks and responsibilities that they usually would not have as they become aware of parental loss of control, socially weakened positions and loss of status. Heightening the stakes in this shift in roles may be cultural norms of enduring personal suffering for the sake of the family, and strong expectations that children do well in school to make sense of the family's sacrifices. All of this occurs in the context of a migration trajectory with traumatic exposures and losses, high levels of stress, novelty and uncertainty. Of course, individual differences in adaptability and family dynamics, including more or less anxious and emotionally unavailable parents (due to their own trauma exposures) all can add to the balance of vulnerability and resilience. However, the post-migration social context also powerfully influences parentchild relationships, family dynamics and community social support. This context includes media representations of migrant communities and public debates about their status. The narrative of resilience within the family may hang on the thin thread of the promise of security and status in a new home. This hope may be fragile, surrounded by a sea of uncertainty and easily overturned by local bureaucracy and global politics.

In addition to these commonly recognised stressors, we need to consider the communicative function of idioms of distress and illness narratives. The social meaning and consequences of somatic symptoms may be more acceptable than their psychological counterparts, which may be invalidated, stigmatised or dismissed. ${ }^{91}$ This contingent shaping of symptoms need not be conscious or strategic-it can occur automatically through subpersonal processes and, hence, be experienced as involuntary. The response from the social environment may reinforce the symptoms, even when others are trying to resolve the affliction, resulting in feedback loops that amplify and maintain symptoms. ${ }^{92}$ The clinical diagnosis and treatment of symptoms themselves may constitute another amplifying loop. Of course, most of these looping effects are part of ordinary symptom experience, identification, labelling and illness narrative formation. When they allow error correction, such feedback loops can serve regulatory, homeostatic, allostatic or adaptive functions. What makes them vicious circles is not only an individual's neurobiological or psychological vulnerability (due to trauma exposure, loss, etc.), but also the precarity of their social situation, the limited scope and resources for actions to restore balance, and the ways in which institutional and social practices of diagnostic labelling and intervention come to serve other powerful interests.

Identifying the feedback loops that regulate or amplify distress can support an integrative BPS approach to assessment and intervention. At each level of organisation, a different process may be recognised and a corresponding intervention devised, with effects on the whole system. For example, in some instances, focusing on bodily symptoms might provide a sense of control by countering less tangible, and at times disembodied or depersonalised mental states marked by anxiety, low mood, fear and uncertainty. Additionally, relatives and parents may be more available, attentive and able respond to a physical symptom than to a panic attack or depression.

Ensuring recovery, however, requires consideration of the broader social meanings of symptoms and diagnoses. Psychiatric diagnostic labels, attributions and explanations can convey a sense of agency and blame for symptoms and behaviours. Hence, diagnoses can become rhetorical resources, tools or weapons in sociopolitical battles at an interpersonal, family, community or other levels, and can serve either to silence or give voice to the unspoken and unheard.

\section{CONCLUSION}

Inquiry into illness experience, whether for clinical or research purposes, is guided by tacit theories of what is at stake for individuals or communities (clinicians and patients alike) drawing from knowledge of biomedical, socioeconomic, moral and political contexts. Hence, any attempt to explain and categorise a collection of symptoms inevitably reflects its proponents' ontological and epistemological commitments. As a result of cognitive-social looping effects, the symptoms themselves and their evolution over time may be shaped by clinicians' viewpoint and explanatory models.

Although contemporary psychiatric research has provided ample evidence of genome-epigenome-brain-environment interactions over the course of development that contribute to the causes, course and outcome of illness, it has proved difficult to integrate this thinking into diagnostic practice. Indeed, the BPS approach, which was the best hope for an integrative theory and approach to practice in clinical medicine, psychiatry and psychosomatics, has been criticised for lack of content and precision. Current scientific claims and marketing hype about 'precision psychiatry' have so far yielded little of use to clinicians.

There are several major reasons why integrative approaches are difficult to realise in medical practice. Cognitively, it is difficult to think about multidimensional interactions and we tend to default to more binary or dichotomous thinking. The affective valence of experience imposes a basic evaluative structure of good/bad, with corresponding behavioural responses of approach/withdraw. This affective level of meaning underwrites more complex moral distinctions based on ascriptions of volition that contrast voluntary actions that lead to events one is responsible for and events that are just accidents. In addition to these cognitive-affective reasons for dualistic thinking, the nature of medical practice also enforces categorical thinking: diagnoses are either applied or not (although 'NYD' - not yet diagnosed-is a common hedge), tests are performed or not, prescriptions are written or not; the whole repertoire of medical care focuses on discrete actions that support algorithms with branching decision trees.

Integrative models may have final common pathways that result in a small set of predictable outcomes, but more often they involve complex system dynamics that are sensitive to initial or boundary conditions, and hence may be difficult or impossible to predict. This means that the basic clinical tasks of diagnosis, prognosis and intervention cannot be one-off but must occur in ongoing interaction with patients allowing constant adjustment. This is a key motivation for the development of person-centred psychiatry ${ }^{93}$ which aims to characterise the unique circumstances and experience of each person and ensure that treatments are tailored to their needs.

In clinical training, this integrative, person-centred approach can be fostered by specific pedagogical strategies, content and experiences, including training in phenomenological psychopathology, systems thinking, structural competency and cultural formulation. ${ }^{94}$ This can equip clinicians with the conceptual models and practical tools needed for comprehensive, multilevel case formulation. Giving priority to this person-centred approach, however, will also require institutional changes that allow clinicians the space and time to practice a more truly integrative medicine. 
The multilevel, ecosocial view we have advanced here suggests why achieving true precision in healthcare will require attention to patients' lived experience, illness narratives and sociocultural context. We need strategies to teach person-centred, integrative thinking to new generations of clinicians and ways to convey this perspective to the wider public so that patients themselves can be effective partners in their care. This is challenging both because our own cognitive apparatus favours binary categories and dualistic thinking and because the social world pushes back with moral dichotomies. Psychosomatic discourse is always inscribed within particular concepts of personhood and agency, which are maintained by circulating narratives, institutions and social niches. Cultural critique of psychosomatic explanation and clinical practice can document the concrete effects of dualistic forms of thought and practice. Engaging with other conceptual systems and forms of practice that coexist with biomedicine can allow us to find ways to conceive of bodies and minds otherwise than the dominant stories that do not capture patients' lived experience, the complex dynamics of illness, or the potential paths to adaptation, healing and recovery.

Contributors Both authors conceived the paper, reviewed the literature, wrote drafts and agreed on the final version.

Funding This study was funded by Government of Canada, Banting Postdoctoral Fellowship (201709BPF-393951-294377).

Competing interests None declared.

Patient consent for publication Not required.

Provenance and peer review Not commissioned; externally peer reviewed.

Open access This is an open access article distributed in accordance with the Creative Commons Attribution Non Commercial (CC BY-NC 4.0) license, which permits others to distribute, remix, adapt, build upon this work non-commercially, and license their derivative works on different terms, provided the original work is properly cited, appropriate credit is given, any changes made indicated, and the use is non-commercial. See: http://creativecommons.org/licenses/by-nc/4.0/.

\section{NOTES}

1. Anna Swir (1996), Talking to My Body (C. Milosz, Translator) (Port Townsend, WA: Copper Canyon Press), p. 62.

2. Deutsch Felix (1959), On the Mysterious Leap from the Mind to the Body: A Workshop Study on the Theory of Conversion. (New York: International Universities Press).

3. See, for example, Levenson J. L (2018) (Ed.). The American Psychiatric Association Publishing Textbook of Psychosomatic Medicine and Consultation-liaison Psychiatry. (Washington, DC: American Psychiatric Pub); Thomas N. Wise (2014) Psychosomatics: past, present and future. Psychotherapy and Psychosomatics, 83(2), 65-69.

4. Laurence J. Kirmayer (1988), "Mind and body as metaphors: hidden values in biomedicine." In Margaret Lock and Deborah Gordon (Eds.) Biomedicine examined. (New York: Springer) 57-93.

5. Mara Buchbinder (2015), All in Your Head: Making Sense of Paediatric Pain. (Berkeley, CA: University of California Press).

6. Marc J. Miresco and Laurence J. Kirmayer (2006). "The persistence of mind-brain dualism in psychiatric reasoning about clinical scenarios." American Journal of Psychiatry, 163(5): 913-918.

7. Kirmayer, "Mind and body as metaphors: hidden values in biomedicine"

8. W. Ross Ashby (1952), Design for a Brain: The Origin of Adaptive Behaviour (London: Chapman Hall); George A Miller et al. (1960), Plans and the structure of behaviour (New York, NY: Henry Holt and Co); William T. Powers (1973), Behaviour: The Control of Perception. (Chicago: Aldine); Norbert Wiener (1961), Cybernetics or Control and Communication in the Animal and the Machine. (Cambridge, MA: MIT Press); for a history and discussion of the close connections to psychiatry among early contributors to cybernetics see: Andrew Pickering (2010), The Cybernetic Brain: Sketches of Another Future (Chicago: University of Chicago Press).

9. Stephen H. Kellert (1993), In the Wake of Chaos: Unpredictable Order in Dynamical Systems (Chicago, IL: University of Chicago Press).

10. George A. Miller et al. (1960), Plans and the structure of behaviour; Norbert Wiener (1961), Cybernetics or Control and Communication in the Animal and the Machine (Cambridge, MA: MIT Press); Alicia Juarrero (1999), Dynamics in Action (Cambridge, MA: MIT Press)
11. The causal efficacy of narrative arises from its role in cognition and its social functions in self-presentation, navigating the social world, and influencing others. See: Daniel D. Hutto (2012), Folk Psychological Narratives: The Sociocultural Basis of Understanding Reasons (Cambridge, MA: MIT Press); Peter Goldie (2012), The Mess Inside: Narrative, Emotion, and the Mind (Oxford: Oxford University Press).

12. lan Hacking (1999), The Social Construction of What? (Cambridge, MA: Harvard University Press); Ian Hacking and Historical Ontology (2004), Historical Ontology (Cambridge, MA: Harvard University Press).

13. Laurence J. Kirmayer and Norman Sartorius (2007), "Cultural models and somatic syndromes." Psychosomatic Medicine, 69(9): 832-840; Jaakko Kuorikoski and Samuli Pöyhönen (2012), "Looping kinds and social mechanisms," Sociological Theory, 30(3):187-205; Serife Tekin (2014), "The missing self in Hacking's looping effects," in Classifying Psychopathology: Mental Kinds and Natural Kinds, ed. Harold Kincaid and Jacquelin A. Sullivan (Cambridge, MA: MIT Press), 227-256.

14. Hacking, Historical Ontology.

15. David J. Chalmers (1995), "Facing up to the problem of consciousness," Journal of Consciousness Studies, 2 (3): 200-219; Jonathan Shear, Jonathan et al., 1999, (Ed.). Explaining Consciousness: The Hard Problem (Cambridge, MA: MIT Press).

16. For consciousness and narration, see: Daniel C. Dennett (2017), Consciousness Explained (Boston: Little, Brown); for consciousness and links to specific brain circuitry that enable arousal, activation, attention, and awareness, see: Christof Koch (2004), The Quest for Consciousness: A Neurobiological Approach (Englewood, CO: Roberts and Company); for consciousness as the overall capacity for multimodal information integration in the brain, see: Gerald Edelman and Giulio Tononi (2008), A Universe of Consciousness: How Matter Becomes Imagination (New York: Basic Books); for consciousness as intrinsic to sensory-perceptual engagements, see: Nicholas Humphrey (2006), Seeing Red: A Study in Consciousness (Cambridge, MA: Harvard University Press).

17. D. H. Rakison and D. Poulin-Dubois (2001), "Developmental origin of the animateinanimate distinction," Psychological Bulletin 127, no. 2: 209.

18. Todd D. Little et al. (2006), "The agentic self: On the nature and origins of personal agency across the lifespan," in Handbook of Personality Development, ed. Daniel K. Mroczek and Todd D. Little (New York: Psychology Press), 61-79.

19. Kirmayer, "Mind and body as metaphors: hidden values in biomedicine."

20. Michael Lambek (1998), "Body and mind in mind, body and mind in body: Some anthropological interventions in a long conversation," in Bodies and persons: Comparative perspectives from Africa and Melanesia, ed. Michael Lambek and Andrew Strathern (New York: Cambridge University Press), 103-123.

21. Lonzozou Kpanake (2018), "Cultural concepts of the person and mental health in Africa," Transcultural Psychiatry 55, no. 2: 198-218.

22. Brigit Obrist and Silvia Büchi (2008). "Stress as an idiom for resilience: health and migration among sub-Saharan Africans in Switzerland." Anthropology \& medicine 15, no. 3: 251-261.

23. Kelly G. Shaver (2012), The attribution of blame: Causality, responsibility, and blameworthiness (New York: Springer).

24. Chloe G. K Atkins (2010), My Imaginary IIIness: A Journey into Uncertainty and Prejudice in Medical Diagnosis (Ithaca, NY: Cornell University Press).

25. K. Looper and Laurence J. Kirmayer (2004). "Perceived stigma in functional somatic syndromes and comparable medical conditions." Journal of Psychosomatic Research 57, no. 4: 373-378.

26. Georg Groddeck (1965), The Book of the It (Orig. pub. 1923, New York: Vintage).

27. Laurence J. Kirmayer, 1999, "Rhetorics of the body: medically unexplained symptoms in sociocultural perspective," in Somatoform Disorders: A Worldwide Perspective, ed. Yutaka Ono, Aleksandar Janca, Masahiro Asai, and Norman Sartorius (Tokyo: Springer), 271-286; Sarah Nettleton (2006), "I just want permission to be ill': towards a sociology of medically unexplained symptoms," Social science \& medicine 62 , no. 5: 1167-1178; Anne van Gils et al, (2016), "Self-help for medically unexplained symptoms: a systematic review and meta-analysis," Psychosomatic medicine 78, no. 6: 728-739.

28. G L Engel (1977), "The need for a new medical model: a challenge for biomedicine," Science 196, no. 4286: 129-136; George L. Engel (1978), "The biopsychosocial model and the education of health professionals," Annals of the New York Academy of Sciences 310, no. 1: 169-181.

29. Michael Saraga, Abraham Fuks, and J. Donald Boudreau (2014). "George Engel's epistemology of clinical practice," Perspectives in biology and medicine 57, no. 4: 482-494

30. Wendell S. Muncie (1959), "The psychobiological approach," in American Handbook of Psychiatry, ed. Silvano Arieti (New York, NY: Basic Books), 1317-1332.

31. Ludwig Von Bertalanffy (1968), General Systems Theory, (New York: Braziller).

32. Engel, "The need for a new medical model: a challenge for biomedicine"; George L. Engel (1997); Kirmayer, "Mind and body as metaphors: hidden values in biomedicine." 
33. R. Frankel et al. (2003). "The Biopsychosocial Approach: Past, Present, Future," The Permanente Journal 8, no. 3 (2004): 75. George L. Engel (1980)

34. S. Nassir Ghaemi (2010), The Rise and Fall of the Biopsychosocial Model: Reconciling Art and Science in Psychiatry (Baltimore, MD: Johns Hopkins University Press).

35. For a portrait of the central role of psychodynamics in U.S. psychiatric training up to the 1970s, see: Donald Light, Becoming Psychiatrists: The Professional Transformation of Self (New York: W.W. Norton, 1980); for an account of the dethronement of psychoanalysis by biological psychiatry from the 1980s onward, see: Tanya M. Luhrmann (2000), Of Two Minds: The Growing Disorder in American Psychiatry (New York: A.A. Knopf)

36. John Read et al. (2009), "Time to abandon the bio-bio-bio model of psychosis: exploring the epigenetic and psychological mechanisms by which adverse life events lead to psychotic symptoms," Epidemiology and Psychiatric Sciences 18, no. 4: 299-310.

37. Thomas R. Insel, 2014, "The NIMH research domain criteria (RDoC) project: precision medicine for psychiatry," American Journal of Psychiatry 171, no. 4: 395-397.

38. For critiques of RDoC, see: Laurence J. Kirmayer and Daina Crafa (2014). "What kind of science for psychiatry?" Frontiers in Human Neuroscience, 8: 435; Joel Paris and Laurence J. Kirmayer (2016), "The National Institute of Mental Health Research Domain Criteria: A bridge too far." The Journal of Nervous and Mental Disease 204, no. 1: 26-32.

39. Nancy C. Andreasen (2007), "DSM and the death of phenomenology in America: an example of unintended consequences," Schizophrenia Bulletin, 33 no. 1 (2006): 108-112.

40. Giovanni Stanghellini and Thomas Fuchs (2013), eds. One century of Karl Jaspers' general psychopathology (Oxford University Press).

41. Laurence J. Kirmayer (2015), "Re-visioning psychiatry: toward an ecology of mind in health and illness," in Re-visioning psychiatry: Cultural phenomenology, critical neuroscience, and global mental health, eds. Laurence J. Kirmayer, Robert Lemelson, and Constance A. Cummings (Cambridge University Press), 622-660.

42. Laurence J. Kirmayer and Maxwell J. D. Ramstead (2017). "Embodiment and Enactment in Cultural Psychiatry," in Embodiment, Enaction, and Culture: Investigating the Constitution of the Shared World, eds. Christoph Durt, Christian Tewes, Thomas Fuchs (Cambridge, MA: MIT Press), 397-422.

43. Andy Clark (2008), Supersizing the mind: Embodiment, action, and cognitive extension (OUP USA); Shaun Gallagher (2006), How the Body Shapes the Mind (Oxford: Clarendon Press); Raymond W. Gibbs Jr (2005), Embodiment and Cognitive Science (New York: Cambridge University Press).

44. Laurence J. Kirmayer, 1992, "The body's insistence on meaning: metaphor as presentation and representation in illness experience," Medical anthropology quarterly 6, no. 4: 323-346.

45. Maxwell J. D. Ramstead, Samuel P. L. Veissière, and Laurence J. Kirmayer (2016), "Cultural affordances: scaffolding local worlds through shared intentionality and regimes of attention," Frontiers in Psychology 7: 1090.

46. Alicia Juarrero (1999), Dynamics in action; Mog Stapleton and T. Froese (2016), "The enactive philosophy of embodiment: From biological foundations of agency to the phenomenology of subjectivity," in Biology and subjectivity: Philosophical Contributions to Non-reductive Neuroscience, eds. Miguel García-Valdecasas, José I. Murillo, and Nathaniel F. Barrett (New York: Springer), 113-129.

47. Bernhard Hommel, 2015, "Action control and the sense of agency," in The Sense of Agency, eds. Patrick Haggard and Baruch Eitam (New York: Oxford University Press), 307-326.

48. Caitlin M Fausey et al. (2010). "Constructing agency: the role of language." Frontiers in Psychology 1: 162; Shaun Gallagher (2012), "Multiple aspects in the sense of agency," New ideas in Psychology 30, no. 1: 15-31.

49. Compare, for example, the following scenarios in which you turn on a light switch because: i) You accidentally fell against the switch (a mechanical response of one's body as an inert object); ii) You were pushed by someone else against the switch (a mechanical response to the actions of another); iii) You always do this when entering the room (habitually, automatically); iv) You felt anxious or uneasy in the dark and more comfortable when the lights are on (as a more or less reflective response to an emotion); v) The room was dark and you wanted to read (as an unreflective, automatic part of a (arger plan); vi) You decided to turn it on ("intentionally"); vii) It is customary to turn it on at that time of day (as an internalised social norm); or, viii) You were told, asked, or ordered to by someone else to turn it on (in response to various forms of social influence or pressure); Each of these scenarios implies a different sort of agency that depends on our understanding and attributions of the origins of action (its cause) and its subsequent control. For a discussion of multiple kinds of control as seen in dissociative phenomena (which may account for conversion symptoms) see: Ernest R. Hilgard (1997), Divided Consciousness: Multiple Controls in Human Thought and Action (New York: John Wiley \& Sons).
50. Tine Jaspers et al. (2009). "Pervasive refusal syndrome as part of the refusalwithdrawal-regression spectrum: critical review of the literature illustrated by a case report," European Child \& Adolescent Psychiatry 18, no. 11: 645; Göran Bodegård (2014), "Comment on the paper "Pervasive Refusal Syndrome (PRS) 21 years on-a reconceptualization and renaming" by Ken Nunn, Bryan Lask and Isabel Owen," European Child and Adolescent Psychiatry, 23, no. 3: 179-181; Karl Sallin et al. (2016). "Resignation syndrome: catatonia? Culture-bound?," Frontiers in Behavioural Neuroscience 10: 7.

51. M Hessle and N. Ahmadi (2006), Asylsökande Barnmed Uppgivenhetssymtom- Trauma, Kultur, Asy/process. SOU 2006:49. Statens Offentliga Utredningar; P. A. Rydelius (2006). Rapport om de.K.Apatiska Barnen. Research Report, No. 25.ISSN1403-607X. Stockholm, Karolinska Institutet.

52. Barn Med Uppgivenhetssyndrom Envägledning för Personal inom Socialtjänstoch Hälso-och Sjukvård (Stockholm: Socialstyrelsen, 2013); WHO, The ICD-10 classification of mental and behavioural disorders: clinical descriptions and diagnostic guidelines, (Geneva: World Health Organization, 1992).

53. For 'lethal mothering' or trauma reenactment see: Göran Bodegård (2005), "Lifethreatening loss of function in refugee children: another expression of pervasive refusal syndrome?" Clinical Child Psychology and Psychiatry, 10, no. 3: 337-350; for the notion of 'resistance through regression' seen as part of a refusal-withdrawalregression spectrum see: Tine Jaspers et al. (2009). "Pervasive refusal syndrome as part of the refusal-withdrawal-regression spectrum: critical review of the literature illustrated by a case report," European Child \& Adolescent Psychiatry 18, no. 11: 645. D M Dhossche et al. (2012), Susan Lynne Thompson and Kenneth Patrick Nunn (1997); For Trauma and Environment see: Hans Peter Söndergaard et al. (2012)

54. Göran Bodegård (2005), "Pervasive loss of function in asylum-seeking children in Sweden." Acta Paediatrica, 94, no. 12, (2005): 1706-1707; Göran Bodegård (2005), "Life-threatening loss of function in refugee children: another expression of pervasive refusal syndrome?"; Liv Lyngå Von Folsach and Edith Montgomery (2006), "Pervasive refusal syndrome among asylum-seeking children," Clinical Child Psychology and Psychiatry 11, no. 3 (2006): 457-473.

55. Von Folsach and Montgomery, "Pervasive refusal syndrome among asylum-seeking children."

56. M Hessle and N. Ahmadi (2006), Asylsökande Barnmed UppgivenhetssymtomTrauma, Kultur, Asylprocess; Karl Sallin et al. (2016). "Resignation syndrome: catatonia? Culture-bound?."

57. lan Hacking (2010), "Pathological withdrawal of refugee children seeking asylum in Sweden," Studies in History and Philosophy of Science Part C: Studies in History and Philosophy of Biological and Biomedical Sciences 41, no. 4: 309-317.

58. Sallin et al., "Resignation syndrome: catatonia? Culture-bound?."

59. Göran Bodegård (2005b), "Life-threatening loss of function in refugee children: another expression of pervasive refusal syndrome?"; Tine Jaspers et al. (2009). "Pervasive refusal syndrome as part of the refusal-withdrawal-regression spectrum."

60. B Aronsson et al. (2009). "Asylum-seeking children with severe loss of activities of daily living: clinical signs and course during rehabilitation," Acta Paediatrica 98, no. 12: 1977-1981.

61. Sallin et al., "Resignation syndrome: catatonia? Culture-bound?."

62. For a discussion of clinical epistemology in psychosomatic diagnosis see: Laurence J. Kirmayer (1994), "Improvisation and authority in illness meaning," Culture, Medicine and Psychiatry 18, no. 2: 183-214.

63. Laurence J. Kirmayer and Norman Sartorius (2007), "Cultural models and somatic syndromes; Jaakko Kuorikoski and Samuli Pöyhönen (2012), "Looping kinds and social mechanisms"; Serife Tekin (2014), "The missing self in Hacking's looping effects."

64. Edward Shorter (1994), From the Mind into the Body: The Cultural Origins of Psychosomatic Symptoms (New York: The Free Press.)

65. Looper and Kirmayer, "Perceived stigma in functional somatic syndromes and comparable medical conditions"; Stephen T. Holgate et al, (2011), "Chronic fatigue syndrome: understanding a complex illness," Nature Reviews Neuroscience 12, no. 9: 539; Catherine Lord and Rebecca M. Jones (2012), "Annual Research Review: Rethinking the classification of autism spectrum disorders," Journal of Child Psychology and Psychiatry 53, no. 5: 490-509; Francisco Ortega (2009), "The cerebral subject and the challenge of neurodiversity," BioSocieties, 4 no. 4: 425-445.

66. B. Lask et al. (1991). "Children with pervasive refusal," Archives of Disease in Childhood 66, no. 7: 866-869; Bodegård, "Comment on the paper

"Pervasive Refusal Syndrome (PRS) 21 years on-a reconceptualization and renaming" by Ken Nunn, Bryan Lask and Isabel Owen"; for further details see: Liv Lyngå Von Folsach and Edith Montgomery (2006), "Pervasive refusal syndrome among asylum-seeking children."

67. Kenneth P. Nunn et al. (2014), "Pervasive refusal syndrome (PRS) 21 years on: a reconceptualisation and a renaming," European Child \& Adolescent Psychiatry 23, no. 3: 163-172.

68. Bodegård, "Pervasive loss of function in asylum-seeking children in Sweden." 
69. M Hessle and N. Ahmadi (2006), Asylsökande Barnmed UppgivenhetssymtomTrauma, Kultur, Asylprocess; Hacking, "Pathological withdrawal of refugee children seeking asylum in Sweden," 312.

70. Hacking, "Pathological withdrawal of refugee children seeking asylum in Sweden,' 310

71. Joel E. Dimsdale et al. (2013). "Somatic symptom disorder: an important change in DSM." Journal of Psychosomatic Research 75, no. 3: 223-228; Oye Gureje (2015), "Classification of somatic syndromes in ICD-11." Current opinion in psychiatry 28, no. 5: 345-349.

72. Göran Bodegård (2014), "Comment on the paper "Pervasive Refusal Syndrome (PRS) 21 years on - a reconceptualization and renaming," 179-181.

73. Daniel D. Hutto (2012), Folk Psychological Narratives: The Sociocultural Basis of Understanding Reasons (Cambridge, MA: MIT Press).

74. Kirmayer, "Resistance, reactance, and reluctance to change: A cognitive attributional approach to strategic interventions," Journal of Cognitive Psychotherapy 4, no. 2: 83-104.

75. Laurence J Kirmayer (2015), "Re-visioning psychiatry: Toward an ecology of mind in health and illness." ; Lucy Yardley (1996), "Reconciling discursive and materialist perspectives on health and illness: A reconstruction of the biopsychosocial approach," Theory \& Psychology 6, no. 3: 485-508.

76. Laurence J Kirmayer and lan Gold (2012), "Critical neuroscience and the limits of reductionism," in Critical Neuroscience: A Handbook of the Social and Cultural Contexts of Neuroscience, eds. Suparna Choudhury and Jan Slaby (Blackwell) 307330; Kirmayer and Ramstead, "Embodiment and Enactment in Cultural Psychiatry," 397-422.

77. See for example Karl Sallin et al. (2016). "Resignation syndrome: catatonia? Culturebound?." ; Anil K. Seth (2013), "Interoceptive inference, emotion, and the embodied self," Trends in cognitive sciences 17, no. 11: 565-573.

78. Kirmayer and Sartorius, "Cultural models and somatic syndromes," 832-840.

79. Ian Hacking (2010), "Pathological withdrawal of refugee children seeking asylum in Sweden," 309-317.lan Hacking (1998)

80. Shorter, From the Mind into the Body: The Cultural Origins of Psychosomatic Symptoms. 81. Kirmayer, "Re-visioning psychiatry: Toward an ecology of mind in health and illness."

82. Karl Friston (2014), "Active inference and agency," Cognitive Neuroscience 5, no. 2: 119-121. doi:10.1080/17588928.2014.905517; Andy Clark (2015), Surfing Uncertainty: Prediction, Action, and the Embodied Mind.

83. Karl Friston (2005), "A theory of cortical responses," Philosophical Transactions of the Royal Society of Lond on B Biological Sciences I. 360: 815 836. doi:10.1098/ rstb.2005.1622; Chris Frith, Making Up the Mind: How the Brain Creates Our Mental World (Oxford: Blackwell). Chris Frith (2007)

84. Karl Friston (2012), "Prediction, perception and agency," International Journal of Psychophysiology 83, no. 2: 248-252.

85. Frederike H. Petzschner et al. (2017)., "Computational psychosomatics and computational psychiatry: Toward a joint framework for differential diagnosis." Biological Psychiatry 82, no. 6: 421-430.

86. Sallin et al., "Resignation syndrome: catatonia? Culture-bound?."

87. Ibid, p13.

88. Manos Tsakiris and De Preester Helena (2018), The Interoceptive Mind: From Homeostasis to Awareness (New York: Oxford University Press).

89. Maxwell J. D. Ramstead, Samuel P. L. Veissière, and Laurence J. Kirmayer (2016), "Cultural affordances: scaffolding local worlds through shared intentionality and regimes of attention," Frontiers in Psychology, 7: 1090.

90. Kirmayer, "Re-visioning psychiatry: Toward an ecology of mind in health and illness"

91. Laurence J. Kirmayer et al. (1998), "Somatization and psychologization: Understanding cultural idioms of distress," in Clinical Methods in Transcultural Psychiatry, ed. Samuel Okpaku (Washington, DC: American Psychiatric Publishing), 233-265.

92. Kirmayer and Sartorius, "Cultural models and somatic syndromes."

93. Juan E. Mezzich et al. (2016). Person Centred Psychiatry (New York: Springer).

94. Ibid; Jonathan M. Metzl and Helena Hansen (2014). "Structural competency: Theorising a new medical engagement with stigma and inequality." Social Science \& Medicine 103: 126-133; Roberto Lewis-Fernández et al. (2015) (eds.) DSM-5 Handbook on the Cultural Formulation Interview. American Psychiatric Pub; Laurence J. Kirmayer (2015), Re-visioning psychiatry. Laurence J. Kirmayer and Ana Gómez-Carrillo (2019)

\section{BIBLIOGRAPHY}

Andreasen, Nancy C. "DSM and the death of phenomenology in America: an example of unintended consequences." Schizophrenia Bulletin 33, no. 1 (2007): 108-12.

Aronsson, B, C. Wiberg, P. Sandstedt, A. Hjern, and Anders Hjern. "Asylum-seeking children with severe loss of activities of daily living: clinical signs and course during rehabilitation." Acta Paediatrica 98, no. 12 (2009): 1977-81.
Ashby, W. Ross. Design for a Brain: The Origin of Adaptive Behaviour. London: Chapman Hall, 1952.

Atkins, Chloe G. K. My Imaginary Illness: A Journey into Uncertainty and Prejudice in Medical Diagnosis. Ithaca, NY: Cornell University Press, 2010.

Bodegård, Göran. "Pervasive loss of function in asylum-seeking children in Sweden." Acta Paediatrica 94, no. 12 (2005a): 1706-7.

. "Life-threatening loss of function in refugee children: another expression of pervasive refusal syndrome?" Clinical Child Psychology and Psychiatry 10, no. 3 (2005b): 337-50.

. "Comment on the paper "Pervasive Refusal Syndrome (PRS) 21 years on-a reconceptualization and renaming" by Ken Nunn, Bryan Lask and Isabel Owen." European Child \& Adolescent Psychiatry 23, no. 3 (2014): 179-81.

Buchbinder, Mara. All in Your Head: Making Sense of Pediatric Pain. Berkeley, CA: University of California Press, 2015.

Chalmers, David J. "Facing up to the problem of consciousness." Journal of Consciousness Studies 2, no. 3 (1995): 200-19.

Clark, Andy. Supersizing the Mind: Embodiment, Action, and Cognitive extension. New York: Oxford University Press, 2008.

Surfing Uncertainty: Prediction, Action, and the Embodied Mind. New York: Oxford University Press, 2015.

Dennett, Daniel C. Consciousness Explained. Boston: Little, Brown, 2017

Dhossche, D. M, C. A. RossL. Stoppelbein. "The role of deprivation, abuse, and trauma in pediatric catatonia without a clear medical cause." Acta Psychiatrica Scandinavica 125, no. 1 (2012): 25-32.

Dimsdale, Joel E, Francis Creed, Javier Escobar, Michael Sharpe, Lawson Wulsin, Arthur Barsky, Sing Lee, Michael R. Irwin, and James Levenson. "Somatic symptom disorder: an important change in DSM." Journal of Psychosomatic Research 75, no. 3 (2013): 223-8.

Edelman, Gerald, and Giulio Tononi. A Universe of Consciousness: How Matter Becomes Imagination. New York: Basic Books, 2008.

Engel, G. L. "The need for a new medical model: a challenge for biomedicine." Science 196, no. 4286 (1977): 129-36

Engel, George L. "The biopsychosocial model and the education of health professionals." Annals of the New York Academy of Sciences 310, no. 1 (1978): 169-81.

. "The clinical application of the biopsychosocial model." American Journal of Psychiatry 137, no. 5 (1980): 535-44.

. "From biomedical to biopsychosocial. being scientific in the human domain." Psychosomatics 38, no. 6 (1997): 521-8.

Fausey, Caitlin M, Bria L. Long, Aya InamoriLera Boroditsky. "Constructing agency: the role of language." Frontiers in Psychology 1 (2010): 162

Felix, Deutsch. ed. On the Mysterious Leap from the Mind to the Body: A Workshop Study on the Theory of Conversion. New York: International Universities Press, 1959.

Frankel, R, T. E, Quill, and S. H, McDaniel. eds. The Biopsychosocial Approach: Past, Present, and Future. Rochester, New York: University Rochester Press, 2003.

Friston, Karl. "A theory of cortical responses." Philosophical Transactions of the Royal Society B: Biological Sciences 360, no. 1456 (2005): 815-36.

. "Prediction, perception and agency." International Journal of Psychophysiology 83, no. 2 (2012): 248-52.

$119-21$.

Frith, Chris. Making Up the Mind: How the Brain Creates Our Mental World. Blackwell: Oxford, 2007.

Gallagher, Shaun. How the Body Shapes the Mind. Clarendon Press: Oxford, 2006. . "Multiple aspects in the sense of agency." New Ideas in Psychology 30, no. 1 (2012): 15-31.

Ghaemi, S. Nassir. The Rise and Fall of the Biopsychosocial Model: Reconciling Art and Science in Psychiatry. Baltimore, MD: Johns Hopkins University Press, 2010.

Gibbs Jr, Raymond W. Embodiment and Cognitive Science. New York: Cambridge University Press, 2005.

Goldie, Peter. The Mess Inside: Narrative, Emotion, and the Mind. Oxford: Oxford University Press, 2012.

Groddeck, Georg. The Book of the It. (Orig. pub. 1923). New York: Vintage, 1965.

Gureje, Oye. "Classification of somatic syndromes in ICD-11." Current Opinion in Psychiatry 28, no. 5 (2015): 345-9.

Hacking, lan. Mad travelers: Reflections on the reality of transient mental illnesses. Charlottesville, VA: University of Virginia Press, 1998.

. The Social Construction of What? Cambridge, MA: Harvard University Press, 1999.

. Historical Ontology. Cambridge, MA: Harvard University Press, 2004. . "Pathological withdrawl of refugee children seeking Asylum in Sweden." Studies in History and Philosophy of Science Part C: Studies in History and Philosophy of Biological and Biomedical Sciences 41, no. 4 (2010): 309-17.

Hessle, MN. Ahmadi. Asylsökande Barnmed Uppgivenhetssymtom-Trauma, Kultur, Asylprocess. SOU 2006:49: Statens Offentliga Utredningar, 2006.

Hilgard, Ernest R. Divided Consciousness: Multiple Controls in Human Thought and Action. New York: John Wiley \& Sons, 1997. 
Holgate, Stephen T, Anthony L Komaroff, Dennis Mangan, and Simon Wessely. "Chronic fatigue syndrome: understanding a complex illness." Nature reviews. Neuroscience 12, no. 9 (2011): 539-44.

Hommel, Bernhard. "Action control and the sense of agency." In: The Sense of Agency, edited by P. Haggard, and B. Eitam, 307-26. New York: Oxford University Press, 2015

Humphrey, Nicholas. Seeing Red: A Study in Consciousness. Cambridge, MA: Harvard University Press, 2006.

Hutto, Daniel D. Folk Psychological Narratives: The Sociocultural Basis of Understanding Reasons. Cambridge, MA: MIT Press, 2012.

Insel, Thomas R. "The NIMH research domain criteria (RDoC) project: precision medicine for psychiatry." American Journal of Psychiatry 171, no. 4 (2014): 395-7.

Jaspers, Tine, G. M. J. Hanssen, Judith A. van der Valk, Johann H. Hanekom, Gijs Th J. van WellJan N. M. Schieveld. "Pervasive refusal syndrome as part of the refusalwithdrawal-regression spectrum: critical review of the literature illustrated by a case report." European Child \& Adolescent Psychiatry 18, no. 11 (2009): 645-51.

Juarrero, Alicia. Dynamics in action. Cambridge, MA: MIT Press, 1999.

Kellert, Stephen H. In the Wake of Chaos: Unpredictable Order in Dynamical Systems. Chicago, L: University of Chicago Press, 1993.

Kirmayer, Laurence J. "The body's Insistence on meaning: metaphor as presentation and representation in illness experience." Medical Anthropology Quarterly 6, no. 4 (1992): $323-46$.

. "Improvisation and authority in illness meaning." Culture, Medicine and Psychiatry 18, no. 2 (1994): 183-214.

. "Mind and body as metaphors: hidden values in biomedicine." In Biomedicine Examined, edited byM. Lock, and D. Gordon, 57-93. New York: Springer, 1988.

- "Resistance, Reactance, and reluctance to change: a cognitive Attributional approach to strategic interventions." Journal of Cognitive Psychotherapy 4, no. 2 (1990): 83-104.

. "Re-visioning psychiatry: Toward an ecology of mind in health and illness." In Re-visioning Psychiatry: Cultural Phenomenology, Critical Neuroscience and Global Mental Health, edited by L. J. Kirmayer, R. Lemelson, and C. Cummings. New York: Cambridge University Press, 2015.

_. "Rhetorics of the body: medically unexplained symptoms in sociocultural perspective." In Somatoform Disorders: A Worldwide Perspective, edited by Y. Ono, A. Janca, M. Asai, and N. Sartorius. Tokyo: Springer, 1999.

Kirmayer, Laurence J, and Daina Crafa. "What kind of science for psychiatry?" Frontiers in Human Neuroscience 8, no. e27407 (2014): 435.

Kirmayer, Laurence J, Trang H. T. Dao, and Andre Smith. "Somatization and psychologization: Understanding cultural idioms of distress." In Clinical Methods in Transcultural Psychiatry (pp. 233-265), edited by S. Okpaku. Washington, DC: American Psychiatric Publishing, 1998.

Kirmayer, Laurence J, and lan Gold. "Critical neuroscience and the limits of reductionism." In Critical Neuroscience: A Handbook of the Social and Cultural Contexts of Neuroscience, (pp. 307-330), edited by S. Choudhury, and J. Slaby: Blackwell, 2012.

Kirmayer, Laurence J, and Ana Gómez-Carrillo. "Culturally responsive clinical psychology and psychiatry: An ecosocial approach." In Cultural Clinical Psychology and PTSD (pp. 3-21), edited by A. Maercker, E. Heim, and L. J. Kirmayer. Hogrefe Verlag, 2019

Kirmayer, Laurence J, Juan E. Mezzich, and C. W. Van Staden. "Health Experience and Values in Person-Centered Assessment and Diagnosis." In Person Centered Psychiatry edited by J. E. Mezzich, M. Botbol, G. N. Christodoulou, C. R. Cloninger, and I. M. Salloum. New York: Springer, 2016

Kirmayer, Laurence J, and Maxwell J. D. Ramstead. "Embodiment and Enactment in Cultural Psychiatry." In Embodiment, Enaction, and Culture: Investigating the Constitution of the Shared World, (pp. 397-422), edited by C. Durt, C. Tewes, and T. Fuchs. Cambridge, MA: MIT Press, 2017

Kirmayer, Laurence J, and Norman Sartorius. "Cultural models and somatic syndromes." Psychosomatic Medicine 69, no. 9 (2007): 832-40.

Koch, Christof. The Quest for Consciousness: A Neurobiological Approach. Englewood, CO: Roberts and Company, 2004.

Kpanake, Lonzozou. "Cultural concepts of the person and mental health in Africa." Transcultural Psychiatry 55, no. 2 (2018): 198-218.

Kuorikoski, Jaakko, and Samuli Pöyhönen. "Looping kinds and social mechanisms." Sociological Theory 30, no. 3 (2012): 187-205.

Lambek, Michael. "Body and mind in mind, body and mind in body: Some anthropological interventions in a long conversation." In Bodies and persons: Comparative perspectives from Africa and Melanesia, (pp, 103-123), edited by M. Lambek, and A. Strathern, 103-23. New York: Cambridge University Press, 1998.

Lask, B, C. Britten, L. Kroll, J. Magagna, and M. Tranter. "Children with pervasive refusal." Archives of Disease in Childhood 66, no. 7 (1991): 866-9.

Levenson, J. L. ed. The American Psychiatric Association Publishing Textbook of Psychosomatic Medicine and Consultation-liaison Psychiatry. Washington, DC: American Psychiatric Pub, 2018.

Lewis-Fernández, Roberto, Neil Krishan, Aggarwal, Ladson, Hinton, Devon E, Hinton, and Laurence J, Kirmayer et al. eds. DSM-5 Handbook on the Cultural Formulation Interview, edited by, American Psychiatric Pub and, 2015.
Little, Todd D., C. R Snyder, and M Wehmeyer. "The agentic self: On the nature and origins of personal agency across the lifespan." In: Handbook of Personality Development, (pp. 6179), edited by D. K, Mroczek, and T. D, Little. New York: Psychology Press, 2006.

Lord, Catherine, and Rebecca M Jones. "Annual research review: re-thinking the classification of autism spectrum disorders." Journal of Child Psychology and Psychiatry 53, no. 5 (2012): 490-509.

Looper, KLaurence J. Kirmayer. "Perceived stigma in functional somatic syndromes and comparable medical conditions." Journal of Psychosomatic Research 57, no. 4 (2004): 373-8.

Luhrmann, Tanya M. Of Two Minds: The Growing Disorder in American Psychiatry. New York: A.A. Knopf, 2000.

Metzl, Jonathan M, and Helena Hansen. "Structural competency: Theorizing a new medical engagement with stigma and inequality." Social Science \& Medicine 103 (2014): 126-33.

Mezzich, Juan E, Michel Botbol, G. N Christodoulou, C. Robert Cloninger, and I. M Salloum. Person Centered Psychiatry. New York: Springer, 2016

Miller, George A., Eugene Galanter, and Karl H Pribram. Plans and the structure of behavior. New York, NY: Henry Holt and Co, 1960

Miresco, Marc J, and Laurence J. Kirmayer. "The persistence of mind-brain dualism in psychiatric Reasoning about clinical scenarios." American Journal of Psychiatry 163, no. 5 (2006): 913-8.

Muncie, Wendell S. "The psychobiological approach." In American Handbook of Psychiatry (pp. 1317-1332), edited by S, Arieti. New York, NY: Basic Books, 1959.

Nettleton, Sarah. "I just want permission to be ill': Towards a sociology of medically unexplained symptoms." Social Science \& Medicine 62, no. 5 (2006): 1167-78.

Nunn, Kenneth P., Bryan Lask, and Isabel Owen. "Pervasive refusal syndrome (PRS) 21 years on: a re-conceptualisation and a renaming." European Child \& Adolescent Psychiatry 23, no. 3 (2014): 163-72.

Obrist, Brigit, and Silvia Büchi. "Stress as an idiom for resilience: health and migration among sub-Saharan Africans in Switzerland." Anthropology \& Medicine 15, no. 3 (2008): 251-61.

Ortega, Francisco. "The cerebral subject and the challenge of Neurodiversity." BioSocieties 4, no. 4 (2009): 425-45.

Paris, Joel, and Laurence J. Kirmayer. "The National Institute of mental health research domain criteria." The Journal of Nervous and Mental Disease 204, no. 1 (2016): 26-32.

Petzschner, Frederike H, Lilian A.E. Weber, Tim Gard, and Klaas E. Stephan. "Computational psychosomatics and computational psychiatry: toward a joint framework for differential diagnosis." Biological Psychiatry 82, no. 6 (2017): 421-30.

Pickering, Andrew. The Cybernetic Brain: Sketches of Another Future. Chicago: University of Chicago Press, 2010

Powers, William T. Behavior: The control of perception. Chicago: Aldine, 1973.

Rakison, D H, and D Poulin-Dubois. "Developmental origin of the animate-inanimate distinction." Psychological Bulletin 127, no. 2 (2001): 209-28.

Ramstead, Maxwell J. D., Samuel P. L. Veissière, and Laurence J. Kirmayer. "Cultural Affordances: scaffolding local worlds through shared Intentionality and regimes of attention." Frontiers in Psychology 7, no. 599 (2016): 1090.

Read, John, Richard P. Bentall, and Roar Fosse. "Time to abandon the bio-bio-bio model of psychosis: exploring the epigenetic and psychological mechanisms by which adverse life events lead to psychotic symptoms." Epidemiology and Psychiatric Sciences 18, no. 4 (2009): 299-310

Rydelius, P.-A. Rapport om de.k.Apatiska Barnen. Research Report, No. 25.ISSN1403607X. Stockholm: Karolinska Institutet, 2006.

Sallin, Karl, Hugo Lagercrantz, Kathinka Evers, Ingemar Engström, Anders Hjern, and Predrag Petrovic. "Resignation syndrome: catatonia? Culture-Bound?" Frontiers in Behavioral Neuroscience 10 (2016): 7.

Saraga, Michael, Abraham Fuks, and J. Donald Boudreau. "George Engel's Epistemology of clinical practice." Perspectives in Biology and Medicine 57, no. 4 (2014): 482-94.

Seth, Anil K. "Interoceptive inference, emotion, and the embodied self." Trends in Cognitive Sciences 17, no. 11 (2013): 565-73.

Shaver, Kelly G. The attribution of blame: Causality, responsibility, and blameworthiness. New York: Springer, 2012.

Shear, Jonathan In. Explaining Consciousness: The Hard Problem, edited byj. Shear. Cambridge, MA: MIT Press, 1999.

Shorter, Edward. From the Mind into the Body: The Cultural Origins of Psychosomatic Symptoms. New York: The Free Press, 1994.

Söndergaard, Hans Peter, Mark M. Kushnir, Bernice Aronsson, Per Sandstedt, and Jonas Bergquist. "Patterns of endogenous steroids in apathetic refugee children are compatible with long-term stress." BMC Research Notes 5, no. 1 (2012): 186.

Stanghellini, Giovanni, and Thomas Fuchs. One century of Karl Jaspers' General Psychopathology, New York: Oxford University Press, 2013.

Stapleton, Mog, and T. Froese. "The enactive philosophy of embodiment: From biological foundations of agency to the phenomenology of subjectivity." In: Biology and subjectivity: Philosophical Contributions to Non-reductive Neuroscience, edited by M. GarcíaValdecasas, J. I. Murillo, and N. F. Barrett, 113-29. New York: Springer, 2016.

Swir, Anna. Talking to My Body (C. Milosz, Translator). Port Townsend, WA: Copper Canyon Press, 1996.

Tekin, Serife. "The missing self in Hacking's looping effects." In: Classifying Psychopathology: Mental Kinds and Natural Kinds (pp. 227-256), edited by H. Kincaid and J. A. Sullivan. Cambridge, MA: MIT Press, 2014. 
Thompson, Susan Lynne, and Kenneth Patrick Nunn. "The pervasive refusal syndrome: the RAHC experience." Clinical Child Psychology and Psychiatry 2, no. 1 (1997): 145-65.

Tsakiris, Manos, and De Preester, Helena. eds. The Interoceptive Mind: From Homeostasis to Awareness. New York: Oxford University Press, 2018.

van Gils, Anne, Robert A. Schoevers, Irma J. Bonvanie, Jeannette M. Gelauff, Annelieke M. Roest, and Judith G. M. Rosmalen. "Self-help for medically unexplained symptoms: a systematic review and meta-analysis." Psychosomatic medicine 78, no. 6 (2016): 728-39.

Von Bertalanffy, Ludwig. General Systems Theory. New York: Braziller, 1968.

Von Folsach, Liv Lyngå, and Edith Montgomery. "Pervasive refusal syndrome among asylum-seeking children." Clinical Child Psychology and Psychiatry 11, no. 3 (2006): 457-73.
Wiener, Norbert. Cybernetics or Control and Communication in the Animal and the Machine. Cambridge, MA: MIT Press, 1961.

Wise, Thomas N. "Psychosomatics: past, present and future." Psychotherapy and Psychosomatics 83, no. 2 (2014): 65-9.

World Health Organization. The ICD-10 classification of mental and behavioural disorders: clinical descriptions and diagnostic guidelines. Geneva: World Health Organization, 1992.

Yardley, Lucy. "Reconciling discursive and materialist perspectives on health and illness: a Reconstruction of the biopsychosocial approach." Theory \& Psychology 6, no. 3 (1996): 485-508. 